\title{
Portable Smart Messages for Ubiquitous Java-enabled Devices *
}

\author{
Nishkam Ravi, Cristian Borcea, Porlin Kang, and Liviu Iftode \\ Department of Computer Science, Rutgers University, Piscataway, NJ 08854, USA \\ \{nravi, borcea, kangp, iftode $\} @$ cs.rutgers.edu
}

\begin{abstract}
Recent advances in wireless technology allow Javaenabled devices, such as Smart Phones and PDAs, to create mobile ad hoc networks, over which distributed applications can be executed. Although Java shields the programmers from the heterogeneity of the hardware platforms, a common middleware architecture is needed to support a cooperative execution environment in these networks. In this paper, we present a portable runtime system for Smart Messages (SMs), a middleware architecture based on execution migration, that we designed and implemented on top of an unmodified Java virtual machine.

To facilitate portability, we have designed a lightweight migration mechanism based on Java bytecode instrumentation. This mechanism is suitable for mobile ad hoc networks where limited bandwidth and mobility impose constraints on the amount of data transferred. The experimental results for applications executed over wireless networks of HP iPAQs demonstrate the feasibility of our portable runtime system.
\end{abstract}

\section{Introduction}

Recently, we have been witnessing a significant growth in the number of Java-enabled wireless devices. Benefiting from a strong support from industry, Smart Phones and PDAs are the most representative Java-enabled devices currently available on the market. These devices are mostly used for local computations or downloading data from web servers and personal computers. Since these devices are equipped with short-range wireless network interfaces (e.g., 802.11, Bluetooth), they can create mobile ad hoc networks which can be programmed to execute distributed applications.

A person may use a program installed on her Smart Phone to book a free cab which is a peer on a network

* This work is supported in part by the NSF under the ITR Grant Number ANI-0121416 of wireless-enabled cabs, without involving any central server/authority. Downloading data from the Internet on handhelds like phones is a common practice. Popular content stored on the Internet may be costly to download over GPRS/3G due to a fee charged by telecommunications providers. A group of mobile device users may cooperate among themselves to download their assigned portion of the content to reduce the overall cost [12]. Other examples include drivers inquiring other cars about the traffic conditions on particular routes, customers paying their bills in restaurants using their PDAs, or peer-to-peer games among ad hoc groups of students in a campus. The lack of a common execution environment, however, precludes the development of such distributed applications on top of mobile ad hoc networks of Java-enabled devices. Although Java shields the programmers from the heterogeneity of the hardware platforms, a simple and flexible middleware architecture is needed to support a cooperative execution environment in these networks.

In this paper, we present a portable runtime system for Smart Messages (SMs) [5] [11], a middleware architecture based on execution migration, that we designed and implemented on top of an unmodified Java virtual machine. An SM is a user-defined distributed application which executes on nodes of interest named by properties and uses explicit execution migration to move between these nodes. Each node has a virtual machine for SM execution and a name-based memory, called tag space. The SMs use the tag space for content-based naming and persistent shared memory. An SM carries the routing code and routes itself at each node in the path toward a node of interest. The SM middleware architecture achieves flexibility in networks of resource constrained devices by letting the applications dynamically discover nodes providing services or content of interest.

SMs represent an attractive alternative to traditional distributed computing based on end-to-end data transfers in mobile ad hoc networks for two main reasons. First, SMs can adapt to highly dynamic network configurations by instructing the routing code to return the control to application when the network conditions change. Since its execution state is already at the same node, the SM can quickly 
adapt to these changes. Second, SMs simplify the deployment of new applications in ad hoc networks. A user can inject SMs at any node in the network, and consequently the SMs can migrate their code to any node that does not have it.

The original implementation of the SM middleware architecture was based on a modified Java virtual machine (Sun's KVM [2]). Having access to the virtual machine (VM) source code, we were able to implement an efficient migration mechanism. Additionally, the entire software needed for SM execution at nodes was implemented inside the VM to improve the overall performance of the system. This implementation, although powerful and efficient, is not portable. Since most of the new ubiquitous devices come with a pre-installed Java VM (and most of the time users do not want to modify the system software on their devices), it would be beneficial to have the SMs running over a portable runtime system implemented on top of an unmodified VM.

The main issue to be dealt with in a pure Java middleware architecture is how to perform migration without having access to the VM internals. The execution state is located inside the VM and is not directly accessible to the external world. To implement a portable runtime system that runs on top of any Java VM (i.e., pure Java code), we have designed a lightweight migration approach based on Java bytecode instrumentation. Our approach is well suited for mobile ad hoc networks where limited bandwidth and mobility impose constraints on the amount of data that can be transferred. The migration mechanism is general enough to be applied to other systems based on execution migration of Java programs.

We have developed a prototype implementation, where all the components of the SM middleware architecture are implemented within a portable runtime system that can run on top of unmodified Java virtual machines. To evaluate this runtime system, we have installed it on HP iPAQs running Sun's CVM virtual machine [1] on top of Linux. The testbed consists of wireless networks of HP iPAQs equipped with 802.11 cards. The experimental results show that the portable SM implementation, although costlier than the original implementation in terms of execution time, is a feasible solution for programming distributed applications over Java-enabled cell-phones or PDAs. The results also show that the overhead generated by the increase in Java bytecode size as a result of the bytecode instrumentation is negligible.

The rest of this paper is organized as follows. Section 2 describes the SM distributed computing model. Section 3 presents the migration mechanism and the SM portable runtime system. In Section 4, we report experimental results. The related work is discussed in Section 5, and the paper concludes in Section 6.

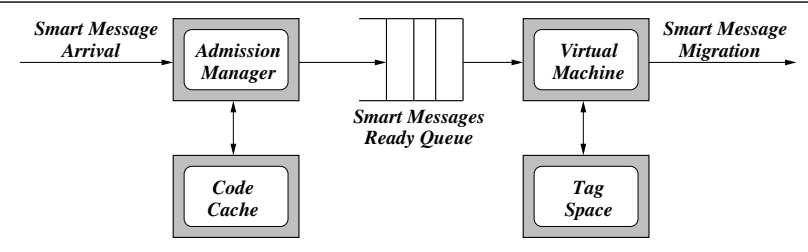

Figure 1. Node Architecture

\section{Distributed Computing with Smart Mes- sages}

A Smart Message (SM) is a user-defined application whose execution is distributed over a series of nodes using execution migration. The nodes on which SMs execute, called nodes of interest, are named by properties and discovered dynamically using application controlled routing. To move between two nodes of interest, an SM calls explicitly for execution migration. An SM consists of code bricks, data bricks (mobile data explicitly identified in the program), and execution control state (e.g., instruction pointers, operand stack pointers). Code bricks are transferred with SMs only if the code is not cached at the destination. An SM can use its code and data bricks to create new, possibly smaller SMs during execution. In this way, an application can eventually generate multiple SMs although it started as a single SM.

\subsection{Node Architecture}

The SM middleware architecture is totally decentralized, with nodes in the network acting as peers. SMs do not make any assumptions about the underlying network configuration, except for a minimal system support provided by nodes. The node architecture is presented in Fig. 1 .

The admission manager is responsible for receiving incoming SMs, deciding whether or not to accept them, and storing them in the SM ready queue. The admission decision is based on a list of resource estimates carried by the SM. The admission manager instructs an accepted SM to transfer only the missing code bricks (i.e., the code bricks that are not stored in the local code cache) and stores them in the cache upon reception.

The virtual machine (VM) offers a hardware abstraction layer for SM execution, which shields the SMs from heterogeneous hardware platforms. The SM execution is nonpreemptive; other SMs can be accepted, but they will not be dispatched for execution before the current SM completes. The execution time is bounded by the estimated running time presented during admission. The non-preemptive scheduling simplifies the implementation of inter-SM synchronization and sharing. 


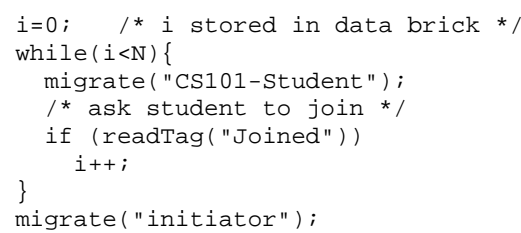

Figure 2. Example of Smart Message Code

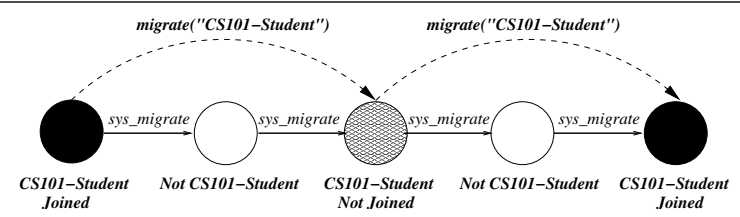

Figure 3. Execution Path for the Smart Message Presented Above

The tag space is a name-based shared memory, persistent across SM executions. It consists of a collection of tags, which essentially are (name, data) pairs used for data exchange among SMs. Special I/O tags are used as an interface to the host OS and I/O system. These I/O tags can be used as an interface to various services provided by nodes. Tags are also used to name the destination of SM migrations as well as to store routing information (routing tags).

\subsection{Smart Messages Example}

To illustrate the SM distributed computing model, let us consider a network of PDAs belonging to students from the same university. At the beginning of each semester the students download on their PDAs an SM that can do two actions: (1) creates a tag for each class the student is registered (e.g., CS101), and (2) helps the student set a group study meeting with other students taking the same class. Using this SM, students need not call other people to set a group study meeting, and even more, they need not know the people registered for that class.

Each time a student wants to set a meeting for a group study, she can inject an SM in the network from her PDA. The goal of this SM is to migrate through the network until it finds $N$ students willing to have a group study for a certain class at a given location and time. Once the group is set, it returns and informs the initiator. This SM is transferred between nodes using short-range wireless network interfaces. For instance, Fig. 2 presents the code for an SM that creates a group study for CS101. Fig. 3 depicts the execution path of this SM over five nodes.
Table 1. Smart Message API

\begin{tabular}{|c|c|}
\hline Category & Primitives \\
\hline Smart Messages & $\begin{array}{l}\text { createSMFromFiles(code_files, data_bricks); } \\
\text { createSM(code_bricks, data_bricks); } \\
\text { spawnSM(); } \\
\text { migrate(tag_names); } \\
\text { sys_migrate(); } \\
\text { blockSM(tag_name, timeout); }\end{array}$ \\
\hline Tag Space & $\begin{array}{l}\text { createTag(tag_name, lifetime, data); } \\
\text { deleteTag(tag_name); } \\
\text { readTag(tag_name); } \\
\text { writeTag(tag_name, data); }\end{array}$ \\
\hline
\end{tabular}

The key operation in the SM programming model is migration, which implements content-based routing using tags. An SM names the nodes of interest by tags, and then calls migrate to route itself to a node that has the desired tags. In our example, migrate ( CS101-Student") routes the SM to students taking CS101 using other PDAs (i.e., belonging to students that do not take CS101) as intermediate nodes. The migrate function uses the sys_migrate primitive for transferring the SM to the next hop. After migration, the SM resumes from the next instruction following the migrate call. It is important to notice that migration is explicit (i.e., the programmer invokes a migrate primitive when needed), and data transferred during a migration is specified by the programmer as data bricks (i.e., in our case, the location and time for the meeting, as well as the variables $i$ and $N$ are transferred as data bricks).

\subsection{Smart Messages API}

The SM API is presented in Table 1. To inject a new SM at a node, users invoke createSMFromFiles with a list of program file names and a list of data bricks. SMs can dynamically create new, possibly smaller SMs by calling createSM or spawnSM. A createSM uses some of the SM's code and data bricks to assemble a new SM and is commonly invoked to build "children" SMs that cooperate with the "parent" SM. An SM may clone itself using spawnSM (similar to the fork system call in Unix).

There are two functions for migration: migrate, and sys_migrate. The migrate function is used by SMs to migrate (over multiple hops) to nodes of interest named by content. The programmers can choose among multiple library implementations of migrate, or they can implement their own versions of this function. To reach these nodes, migrate implements content-based routing algorithms using sys_migrate and routing tags ${ }^{1}$. The sys_migrate primitive implements the protocol for one-hop migration; it captures the execution state and transfers the SM to the next

1 More details about the SM self-routing mechanism are presented in [4] 


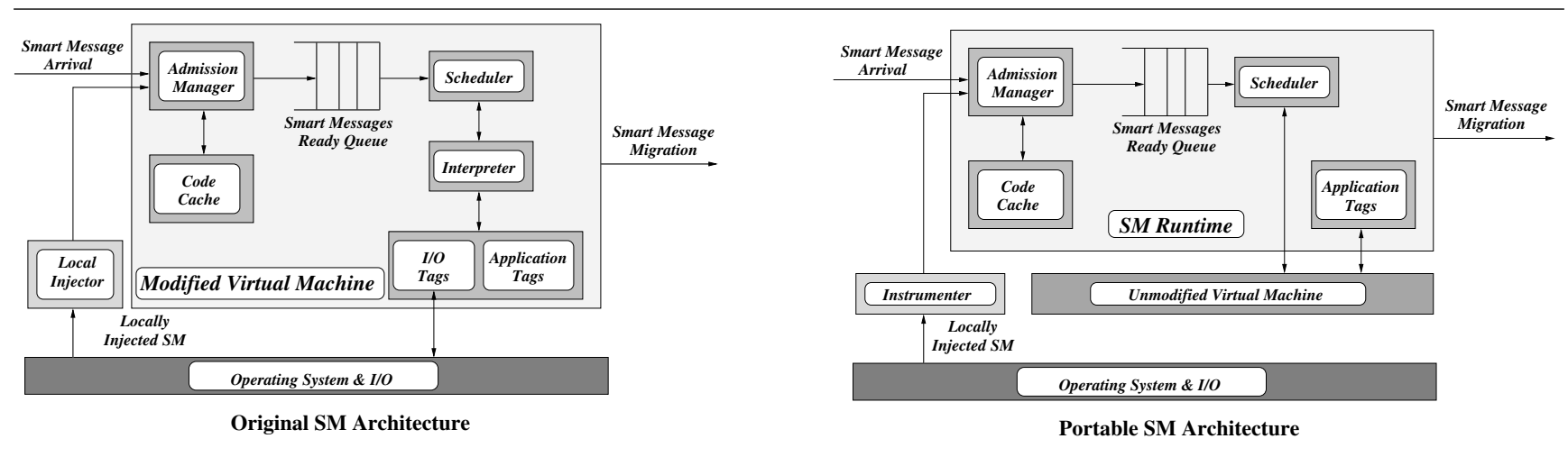

Figure 4. Comparison of the Two Smart Message Architectures

hop. The VM at destination resumes the SM from the instruction following sys_migrate.

An SM can create, delete, or access application tags. The tags are accessed subject to authorization [11]. The same interface is used to access the I/O tags: SMs can issue commands to I/O devices by writing into I/O tags, or can get $\mathrm{I} / \mathrm{O}$ data by reading from I/O tags (an SM cannot create or delete I/O tags).

An SM can invoke blockSM to block on a tag until another SM performs a write on that tag. A blocked SM yields the processor; the VM inserts it into a wait queue associated with the tag. When the VM unblocks an SM, it removes that SM from the wait queue and inserts it back in the SM ready queue. To prevent infinite blocking of SMs, blockSM has a timeout as parameter; if no write operation takes place within this timeout, the SM is unblocked.

\section{Portable Smart Messages}

The original SM implementation was achieved by modifying Sun's Java KVM [2]. The whole architecture, presented in the left part of Fig. 4, was implemented inside the VM because of the need for VM support in capturing the execution state and restoring it at destination to resume the execution. This implementation, although powerful and efficient, is not portable. Since most of the new ubiquitous devices come with a pre-installed Java VM (and most of the time users do not want to modify the system software on their devices), we have designed and implemented a portable SM runtime system (i.e., implemented completely in Java).

The main issue to be solved in a pure Java implementation is how to perform migration without having access to VM internals to capture and restore the execution state. The execution state is located inside the VM and is not directly accessible to the external world.

In order to provide migration without modifying the VM, we have designed a simple and efficient mechanism for cap- turing and restoring the execution state by incorporating all the necessary operations in the SM itself. The heart of our approach lies in instrumenting the SM bytecode in such a way that the SM can save its state before migration and restore it before resumption with a minimal overhead. Using this mechanism, the state is encoded in the code bricks and data bricks, and no explicit state information is shipped.

The right part of Fig. 4 presents our pure Java architecture. Unlike the original architecture which was implemented inside the VM (i.e., for efficiency), the architecture for portable SMs is implemented on top of the VM as a runtime system.

\subsection{Migration}

In the following, we present our migration mechanism, which is generally applicable to any system based on execution migration for Java programs. To migrate an SM, we need to migrate its code bricks, data bricks, and execution control state. The code bricks of SMs are Java class files, and data bricks are Java objects. We use the Java reflection mechanism for loading the classes dynamically at the destination node. The Java serialization mechanism is used to marshal/unmarshal the data bricks across migrations. Since SMs do not use local variables across migrations (i.e., the programmers have to include any data that they need across migrations in the data bricks), object deserialization works fine to restore the values of all objects and variables. The main problem that needs to be solved is how to capture and restore the execution control state (i.e., located inside the VM), which consists of the instruction pointer and the method call stack.

Our solution is to instrument the SM bytecode in such a way that SMs can capture and restore their own runtime stack before resuming their normal execution at destination. There are numerous reasons for choosing bytecode transformation [17, 18] over source code transformation [8]. First, source code transformation does not provide fine-grained 
control as provided by a bytecode transformation (e.g., the lack of goto statement in Java, the difficulty of instrumenting compound statements). Second, instrumenting a loop in source code requires the loop to be unfolded in order to preserve correct execution semantics. Third, instrumenting the source code causes the corresponding bytecode to blow up, and therefore, incurs heavy overheads.

We introduce the term critical method to refer to any method that can directly or indirectly invoke sys_migrate or blockSM. These two methods are the only methods that can lead to capturing and restoring the execution control state. Therefore, only critical methods need to be instrumented. Since a migration (or block) happens at the end of a method call chain, the instrumenter has to detect all the methods from which sys_migrate (or blockSM) is statically reachable in order to recognize critical methods. To simplify the exposition throughout this section, we will refer only to migration.

\subsubsection{Bytecode Instrumentation for Capturing and} Restoring the Execution Control State. Our bytecode instrumenter adds an integer array ip [length] to every class, where length is the number of methods in that class. An element $i p[i]$ is used as a pseudo instruction pointer for the ith method. The code of a critical method is divided into code regions separated by critical method invocations. A critical method invocation marks the end of a code region and the beginning of another new code region. The value of $i p[i]$ is incremented only before a critical method invocation, and hence, serves as a pointer to the boundaries between code regions. At the time of resumption, the value of $i p[i]$ also serves as a pointer to the last statement executed inside the $i t h$ method of the class.

The last statement executed inside a critical method before a migration is always a critical method invocation (i.e., either directly a sys_migrate call or a chain of method invocations that ends with a sys_migrate). This is the reason why incrementing the value of $i p[i]$ only before critical method invocations is sufficient. The value of $i p[i]$ can be used during resumption to locate the last method invocation made from method $i$ before migration. Since every object has a unique $i p$ associated with it, ip is carried over as a part of data bricks and restored during deserialization.

During resumption, each SM starts its execution from the beginning of the $\operatorname{run}()$ method of the main class (i.e., SMs execute as Java threads). The instrumenter introduces a switch statement at the beginning of every critical method to redirect the instruction pointer, based on the value of $i p[i]$, to the last statement executed before migration. Hence, the code already executed is skipped. For every method other than the one that directly invoked sys_migrate, this will result in an invocation of the method that was adjacent to this method in the runtime stack before migration. As a consequence, the runtime stack is recreated. The $i p[i]$ of the method that directly invoked sys_migrate serves as a pointer to the statement immediately following the sys_migrate call.

An SM is said to be in resumption mode when it is recreating the runtime stack. To differentiate between resumption mode and normal execution, the instrumenter adds a global flag: resumption. This flag is important for preserving the correct execution semantics. Its purpose is to activate or deactivate the switch statement introduced by the instrumenter at the beginning of each critical method depending on whether the SM is undergoing normal execution or is in resumption mode. If the SM is resuming, it is necessary to execute the switch statement in order to skip the already executed code. If the SM is undergoing normal execution, it is necessary to ignore the value of $i p[i]$ to ensure that a method invocation is not influenced by this value (i.e., $i p[i]$ might be non-zero due to an earlier invocation of the same method). The resumption flag of the SM is set by the system before the SM is migrated and reset by the SM itself once the SM has reconstructed the method call stack, at which point normal execution of the SM begins. To achieve this, the resumption flag is reset after every statement containing a call to sys_migrate.

Fig. 5 and 6 illustrate the transformation done by the bytecode instrumenter. Although the transformation is done on the bytecode, for the sake of simplicity, we show a higher level transformation on the corresponding Java pseudo-code. In the example, class $A$ has four methods, excluding the constructor. Let us assume that Method1, Method2, and Method4 are critical methods (i.e., they can directly or indirectly invoke sys_migrate or blockSM), while Method3 is not a critical method. We present the bytecode instrumentation only for Method1, but similar transformations take place on the other critical methods (Method 2 and Method4 in this case) as well. As Method 3 according to our assumption is not a critical method, it is not instrumented.

Since class $A$ has five methods including the constructor, $i p$ is declared as an array of length five. We initialize this array in the $<$ init $>$ method which is internal to the bytecode and is invoked every time a new object of the class is created. Given that Method 1 has four invocations to critical methods (two indirect, and two direct), its code is divided into five code regions labeled from 0 to 4 . The value of $i p[1]$ is incremented before every invocation to a critical method. For instance, $i p[1]$ is incremented before an invocation to Method2, but not before an invocation to Method 3 which is not a critical method.

Suppose Method1 had called sys_migrate before migration, the value of $i p[1]$ would be 3 . When the SM resumes execution at the destination node and enters Method 1 , the instruction pointer would be redirected to $l a$ - 


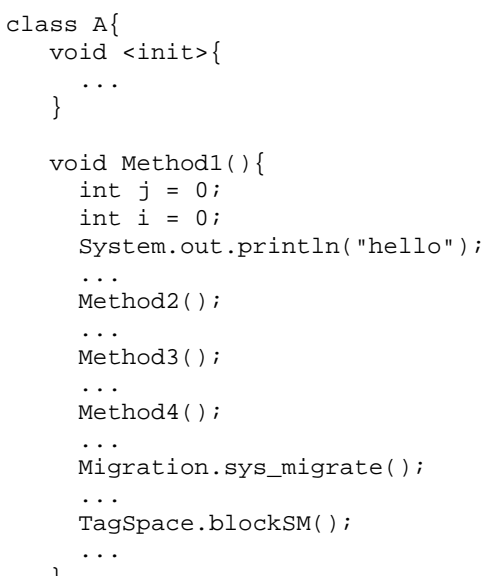

Figure 5. Pseudo-code before Instrumentation

bel 3 by virtue of the switch statement; from this point on, normal execution of the SM begins. If on the other hand Method4 had called sys_migrate, then the value of $i p[1]$ would be 2 . When the SM enters Method 1 after resuming at the destination node, the instruction pointer would be redirected to label 2 which contains a call to Method4, thereby skipping the already executed code in Method 1 and recreating the runtime stack.

This example also shows how the resumption flag is used. If the flag is set to false, the execution of the methods starts from the beginning. Otherwise, it starts with the code region pointed to by $i p[1]$. As soon as the SM recreates the stack, the resumption flag is reset by the SM itself. This ensures that any future invocation to Method 1 or any other critical method will not be affected by the value of $i p$. Note that the resumption flag is local to an SM, but global to all the classes that constitute that SM.

Fig. 7 briefly demonstrates the working of our instrumentation scheme. The upper part of the figure gives a pictorial view of $i p$ in three critical methods at the time of migration. The arrows in the lower part of the figure show the control flow of the SM from the time of execution resumption at the destination until the method stack is recreated.

3.1.2. Bytecode Instrumentation for Suspending a Smart Message. In the original SM implementation, SMs were VM-level threads controlled internally by the VM. In the current implementation, SMs are Java threads, and therefore, the control over SMs is theoretically limited to the amount of control offered by the Java Thread API. When an SM migrates or blocks on a tag, the corre-

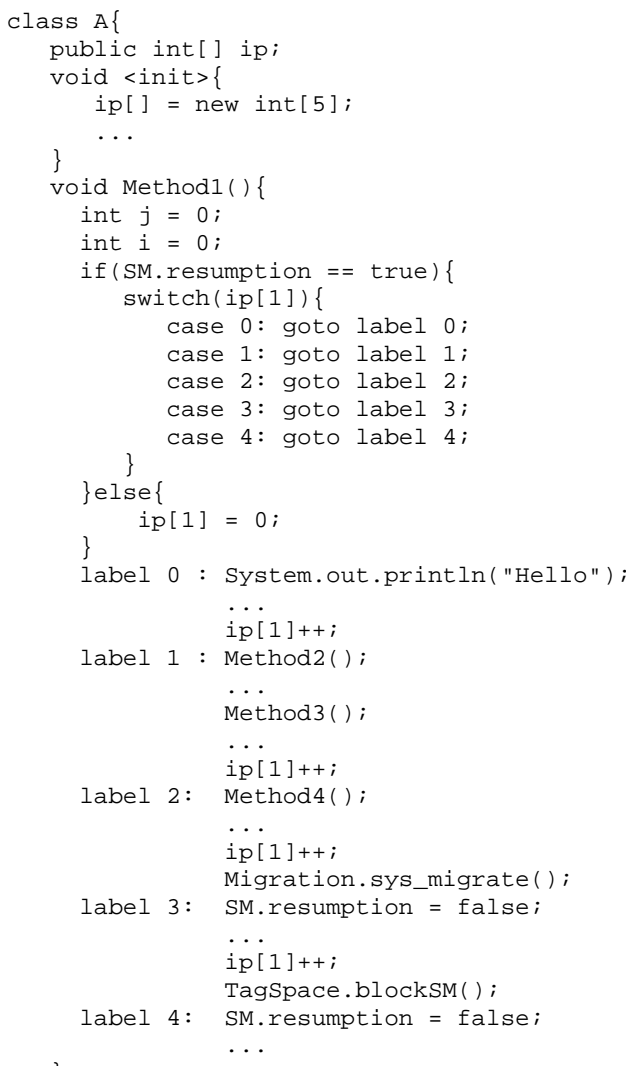

Figure 6. Pseudo-code after Instrumentation

sponding Java thread has to be stopped. The stop () method of the Java Thread class has been deprecated as it was deadlock-prone. In the absence of a direct way of stopping a Java thread, we have used the Java exception mechanism and bytecode transformations.

For each sys_migrate or blockSM call, a StopException (a class that extends the RuntimeException) is thrown. To ensure that this exception is not caught until it reaches the bottom of the stack, every try-catch block is instrumented to re-throw the StopException if it happens to catch it. We ensure that the run() method has a try-catch block that catches this exception and consequently finishes the thread's execution. Using a RuntimeException instead of a regular Exception has the advantage that the method signatures do not have to be modified to include a throws clause.

\subsection{Tag Space}

As mentioned in Section 2, the tag space contains two types of tags: application tags and I/O tags. Since the im- 

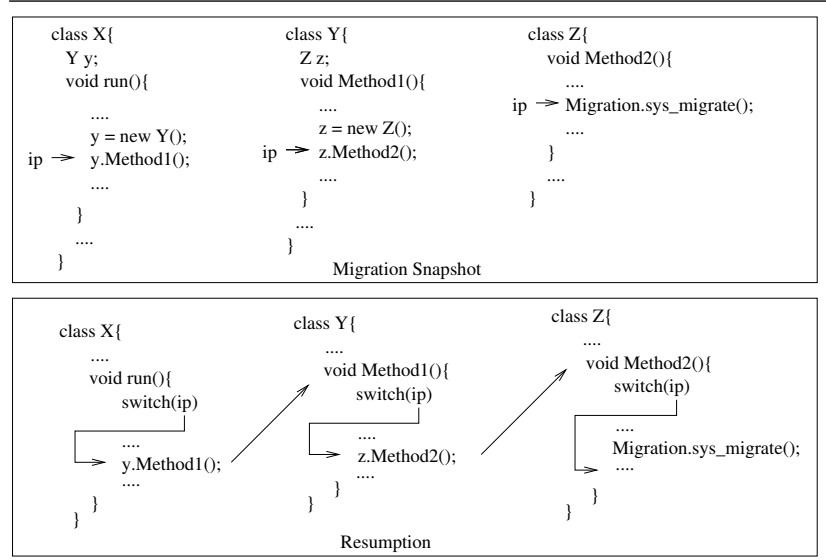
Figure 7. Example of Resuming the Execu-
tion after Migration

plementation of I/O tags is platform dependent, the portable runtime system implements only application tags. These tags are Java objects which can be created, deleted, read from, or written into by SMs.

An SM can also block on a tag for a certain period of time. To implement a timeout mechanism, we use Java's built-in scheduler (i.e., provided by the Scheduler class), which makes the SM ready for scheduling after the timeout. Using Java's Scheduler class avoids a polling timer thread that would otherwise be required to implement the timeout mechanism. Commonly, a blocked SM is woken up by the interpreter when the tag is written by another SM. Each time an SM blocks on a tag, its corresponding Java thread is terminated through the thread stopping mechanism described in Section 3.1.2. Each time an SM is unblocked (and consequently dispatched for execution), a new Java thread is created for it.

\subsection{Code Cache}

We exploit Java's classloader to implement the code cache. The Java reflection mechanism is used to load a class representing a code brick. In the process, a new Class instance of the corresponding class is created. The classloader will not unload the class as long as there is a live reference to the Class instance. References to the cached classes are stored such that these classes are not unloaded by the classloader. When the caching policy chooses a class for eviction, we just remove the stored reference for that class.

\subsection{Scheduler}

The SM scheduler ${ }^{2}$ is implemented as a Java thread that extracts an SM from the ready queue in FIFO order, dispatches it for execution as a Java thread, and goes to sleep. When the SM completes its execution, it wakes up the scheduler using the Java's thread synchronization mechanism.

\subsection{Limitations}

As in the traditional SM architecture, the portable SM architecture does not allow the use of local variables across migrations (they can be used locally, though). Consequently, a local variable cannot occur in two different code regions. All the variables that need to be used across migrations have to be declared as global variables (i.e., they become part of the data bricks). If the programmer wants to use a local variable in two or more code regions, the local variable should be declared and initialized before the beginning of the first code region of that method. This is necessary to satisfy the bytecode verifier.

The lightweight instrumentation scheme does not support recursion across migrations; recursion, however, can be used locally. Our instrumentation scheme relies on the assumption that only one instance of a method is present inside the runtime stack at the time of migration. It should be noted, however, that lack of local variables or recursion across migrations does not compromise the programming model at all.

I/O tags imply coupling the SM runtime system with the OS. In order to make SMs portable, we had to eliminate these tags. The result is a loss of power in the new model, but it can be compensated by various profiles and JSRs provided by J2ME [2] for interacting with the OS or the network. These profiles/JSRs provide Java API for interacting with the OS or the network, thereby hiding the underlying implementation. For instance, the MIDP profile hides the network protocols from the user, provides a generic method of connecting with other devices, and is able to store data persistently without referring to the file system. Another example is the Bluetooth API (JSR 82) which allows connectivity through Bluetooth.

The security issues related to the SM architecture are presented in [11]. Our current implementation does not address these issues. We are in the process of converging upon the correct approach for dealing with the security issues that come as a byproduct of designing a middleware for mobile agents and mobile ad-hoc networks. For cell-phones, which

2 Note that the SM scheduler is a component of the SM architecture and is different from Java's built-in scheduler mentioned in Section 3.2. 
Table 2. Increase in Bytecode Size Due to Instrumentation

\begin{tabular}{|l|c|}
\hline Unmodified Bytecode(KB) & Modified Bytecode(KB) \\
\hline \hline 1084 & 1122 \\
\hline 1230 & 1266 \\
\hline 1527 & 1564 \\
\hline 2330 & 2395 \\
\hline
\end{tabular}

have GPRS connectivity in addition to short-range wireless connectivity, the code could be downloaded from a trusted web server and stored in the code cache either statically or dynamically. This adds a level of security in that it avoids the need of fetching code from an untrusted peer.

\section{Evaluation}

Our goals in conducting the experimental evaluation for the portable SMs were three-fold: (1) quantify the impact of bytecode instrumentation on the SM size, (2) compare the costs for the basic SM operations between our portable architecture and the original SM architecture, and (3) execute a simple application over both architectures in order to compare the completion time.

We use Soot1.2.5 [3] to do the off-line bytecode instrumentation. Table 2 shows the increase in the bytecode size as a result of instrumenting four of our SM test cases. On average, we observe an increase of $2.9 \%$ in the bytecode size which is negligible compared to existing approaches (see Section 5 for details).

We have tested the portable SMs on J2ME CDC platform which uses CVM as the virtual machine. CDC's Personal Profile is the upcoming replacement for Personal Java which is currently used in cell-phones. We have used the reference implementation of CDC's Foundation Profile which is upward compatible with both Personal Profile and Personal Java. Foundation Profile is widely used on PDAs. Our testbed consists of HP iPAQs running Linux. Each iPAQ contains a StrongARM 206MHz processor, 32MB flash memory, and 64MB RAM. For communication we use 802.11b PC cards.

Tables 3 and 4 compare the cost of migration between the portable SM architecture and the original architecture. Table 3 shows the variation of the single-hop round-trip time for an SM as a function of the code brick size for the two cases: (1) when the code is cached (2) when the code is not cached (the data brick size is negligible in this experiment). The relative gain due to caching is more significant for the original implementation; while the original implementation always checks if the code is cached at destination (even when caching is turned off), the portable SM implementation does not perform this check when caching is
Table 3. Effect of Code Brick Size on SingleHop Round-Trip Time

\begin{tabular}{|l|c|c|c|c|}
\hline \multirow{2}{*}{ Size(Bytes) } & \multicolumn{4}{|c|}{ Round-Trip Time(ms) } \\
\cline { 2 - 5 } & \multicolumn{2}{|c|}{ Portable SM } & \multicolumn{2}{c|}{ Original SM } \\
\cline { 2 - 5 } & Uncached & Cached & Uncached & Cached \\
\hline \hline 2322 & 126 & 124 & 56 & 23 \\
\hline 3456 & 150 & 124 & 63 & 23 \\
\hline 4454 & 155 & 124 & 69 & 23 \\
\hline 8510 & 165 & 124 & 91 & 23 \\
\hline
\end{tabular}

\section{Table 4. Effect of Data Brick Size on Single- Hop Round-Trip Time}

\begin{tabular}{|l|c|c|}
\hline \multirow{2}{*}{ Size(Bytes) } & \multicolumn{2}{|c|}{ Round-Trip Time(ms) } \\
\cline { 2 - 3 } & Portable SM & Original SM \\
\hline \hline 2088 & 177 & 29 \\
\hline 4056 & 196 & 38 \\
\hline 8010 & 234 & 57 \\
\hline 16010 & 301 & 88 \\
\hline
\end{tabular}

turned off, thereby saving on communication costs. Table 4 shows the variation of the single-hop round-trip time of an $\mathrm{SM}$ as the size of data bricks varies from $2 \mathrm{~KB}$ to $16 \mathrm{~KB}$ (with code cached). Table 5 shows the cost of tag space operations.

We have implemented and evaluated the Student Study Group application described in Section 2. Table 6 shows the time taken to find $N$ students for a group study and return to the initiator. We have executed this application over an ad hoc network consisting of 8 HP iPAQs, while varying $N$ from 1 to 5 (the nodes of interest have been distributed uniformly in the network). The results indicate that our architecture yields a completion time greater by as much as 3.7 times. The absolute numbers, however, demonstrate that the SM over the portable architecture can still complete between $4.09 \mathrm{~s}$ and $6.33 \mathrm{~s}$ when the code is cached. We consider this time reasonable for mobile ad hoc networks composed of resource constrained devices. The effect of code cache is not very significant for this application because of the unavoidable contention encountered in wireless networks, coupled with our on-demand content-based routing which involves many broadcast messages in the network.

To summarize our results, we have found out that our implementation performs approximately 2 to 3.7 times slower than the original implementation. However, this is the price paid for the ability to inject a new distributed application anytime, anywhere on a Java-enabled device without modifying the system software. We believe that the main reason for these results, besides the fact that Java is inherently slower than $\mathrm{C}$, is that we use an un-optimized vir- 
Table 5. Cost of Tag Space Operations

\begin{tabular}{|l|c|c|}
\hline \multirow{2}{*}{ Operation } & \multicolumn{2}{|c|}{ Time $(\mu \mathrm{s})$} \\
\cline { 2 - 3 } & Portable SM & Original SM \\
\hline \hline readTag & 78 & 21 \\
\hline createTag & 89 & 43 \\
\hline writeTag & 71 & 32 \\
\hline deleteTag & 98 & 56 \\
\hline
\end{tabular}

Table 6. Completion Time for the Student Group Study Application, Varying the Number of Students, $N$, from 1 to 5

\begin{tabular}{|c|c|c|c|c|}
\hline \multirow{2}{*}{$\mathrm{N}$} & \multicolumn{4}{|c|}{ Completion Time(ms) } \\
\cline { 2 - 5 } & \multicolumn{2}{|c|}{ Portable SM } & \multicolumn{2}{c|}{ Original SM } \\
\cline { 2 - 5 } & Uncached & Cached & Uncached & Cached \\
\hline \hline 1 & 4527 & 4093 & 1284 & 1102 \\
\hline 2 & 5212 & 5031 & 1944 & 1783 \\
\hline 3 & 5604 & 5308 & 2036 & 1968 \\
\hline 4 & 6358 & 6012 & 2157 & 1985 \\
\hline 5 & 7863 & 6339 & 2198 & 2148 \\
\hline
\end{tabular}

tual machine (Java CVM based on x86/PC Linux development), while the original implementation uses a virtual machine (Java KVM) designed specifically for resource constrained devices. To quantify the impact of the VM, we plan to run our prototype on the optimized, commercially available CVM. Such an experiment will help us evaluate more accurately the cost of portability (i.e., the cost of implementing the SM architecture as a runtime system on top of unmodified Java VM versus the cost of implementing it within the VM).

\section{Related Work}

Smart Messages (SMs) share the idea of code migration with mobile agents [13,9], and active networks [7, 14].

Similar to a mobile agent, an SM may be viewed as a task that explicitly migrates from node to node and executes on nodes of interest. Unlike mobile agents, SMs are defined to be responsible for their own routing in a network. This feature allows SMs to adapt quickly to changes that may occur both in the network topology and the availability of resources at nodes. A mobile agent names nodes by fixed addresses and commonly knows the network configuration a priori, while an SM names nodes by content and discovers the network configuration dynamically. Furthermore, the SM system architecture defines a node architecture suitable for resource constrained devices.

SMs differ from active networks (AN) in several key features. A first difference comes from the problems they try to solve: AN target improved performance for end-to-end data transfers in relatively stable networks, while SMs help the development of distributed applications on top of a new computing infrastructure which is significantly under-used due to the lack of programmability support. Unlike AN, we define a computing model whereby several SMs can cooperate, exchange data, and synchronize with each other through the tag space. In terms of migration, AN do not transfer the execution state from node to node whereas the SM model does.

To implement execution migration (i.e., transfer of the execution state), two approaches can be used: VM-based or compiler-based. The first approach implies designing new VMs or modifying existing ones to support the capturing and restoring of the execution state. The second approach works for unmodified VMs, but it involves either a modified compiler, or other tools that insert new pieces of code in the source code or directly in the executable program in order to capture and restore the execution state. In the following, we discuss several systems that transfer the execution state for Java programs.

Similar to the original SM implementation, a number of systems $[16,15,6]$ have modified the Java VM (JVM) to provide the required state capturing and restoring. Unlike SMs which were designed specifically for networks of resource constrained devices, these systems are too heavy for devices such as cell-phones or PDAs.

Funfrocken [8] implements transparent migration using a source code transformation mechanism (pre-processor). Capturing the method stacks is done within exception handlers. When a program needs to migrate, an exception is thrown, and in every method, an exception handler is instrumented to save the state of the method stack by creating a new state object for it. The major difference between our approach and this system is in terms of amount of data sent through the network. Using source code transformation, this system increases the size of the bytecode with with as much as $470 \%$. Our bytecode instrumenter increases the size of the bytecode only with as much as $3 \%$. Additionally, this system transfers a significant amount of meta-data for the state objects. Other disadvantages of this system compared to ours include changes of the signatures for all methods to accept a state object as a parameter, and the need for recompilation of all classes (i.e., the complete source code should be available).

Sakamoto et al [17] implement migration using a bytecode transformation scheme that does bytecode verification. Their approach is similar to Funfrocken's in the sense that they also create a state object for every method frame on the stack by using the exception handling mechanism, and therefore, incur comparable growth in the code size. Truyen et al [18] have an implementation that also does bytecode transformation by using the bytecode verification mechanism. Unlike the two previously mentioned ap- 
proaches, they use return and if instructions to roll back the stack when they are creating state objects for every method on the stack which leads to more degradation in performance. Additionally, in order to target multi-threaded environments, they define their own thread-framework: Tasks have to be used instead of threads, and a separate scheduler has been implemented. Although expensive, this approach works well for multi-threaded environments.

The main difference between our approach and the above mentioned bytecode transformation approaches is that we manage to capture and restore execution state without iterating through the runtime stack and creating state objects for every method instance on the stack. By assuming no use of recursion and local variables across migrations, we have been able to devise a lightweight migration approach suitable for embedded systems, without compromising the programming model.

\section{Conclusions and Future Work}

The contribution of this paper is two-fold. First, we have presented a portable middleware architecture for ubiquitous Java-enabled devices which opens up many possibilities for user-defined distributed applications over ad hoc networks composed of Smart Phones or PDAs. Second, we have presented a lightweight execution migration mechanism based on Java Bytecode instrumentation which is suitable for mobile ad hoc networks where bandwidth and mobility impose constraints on the amount of data that can be transferred. The experiments conducted over a mobile ad hoc network of HP iPAQs demonstrate the feasibility of our approach.

We are in the process of porting Smart Messages over Smart Phones [10]. Smart Phones are equipped with both long-range wireless connectivity (GPRS/3G) and shortrange wireless connectivity (Bluetooth/IrDA). We intend to utilize this dual connectivity to provide a security framework for Smart Messages. Code bricks could now be downloaded/authenticated by connecting to a trusted web-server over GPRS/3G.

\section{References}

[1] Java 2 Platform Micro Edition (J2ME), http://java.sun.com/j2me/.

[2] K Virtual Machine. http://java.sun.com/products/cldc/.

[3] Soot: A Java Optimization Framework, http://www.sable.mcgill.ca/soot/.

[4] C. Borcea, C. Intanagonwiwat, A. Saxena, and L. Iftode. Self-Routing in Pervasive Computing Environments using Smart Messages. In Proceedings of the 1st IEEE International Conference on Pervasive Computing and Communications (PerCom 2003), pages 87-96, Dallas-Fort Worth, TX, March 2003.
[5] C. Borcea, D. Iyer, P. Kang, A. Saxena, and L. Iftode. Cooperative Computing for Distributed Embedded Systems. In Proceedings of the 22nd International Conference on Distributed Computing Systems (ICDCS 2002), pages 227-236, Vienna, Austria, July 2002.

[6] S. Bouchenak and D. Hagimont. Pickling threads state in the java system. In Proceedings of the Technology of ObjectOriented Languages and Systems (TOOLS 33), page 22. IEEE Computer Society, 2000.

[7] D. Wetherall. Active Network Vision Reality: Lessons from a Capsule-based System. In Proceedings of the 17th ACM Symposium on Operating Systems Principles (SOSP 1999), pages 64-79, Charleston, SC, December 1999. ACM Press, New York, NY.

[8] S. Funfrocken. Transparent Migration of Java-Based Mobile Agents. In Mobile Agents, pages 26-37, 1998.

[9] R. Gray, G. Cybenko, D. Kotz, and D. Rus. Mobile Agents: Motivations and State of the Art. In J. Bradshaw, editor, Handbook of Agent Technology. AAAI/MIT Press, 2002.

[10] L. Iftode, C. Borcea, N. Ravi, P. Kang, and P. Zhou. Smart phone: An embedded system for universal interactions. In Proceedings of the tenth International Workshop on $\mathrm{Fu}$ ture Trends in Distributed Computing Systems, pages 88-94, 2004.

[11] P. Kang, C. Borcea, G. Xu, A. Saxena, U. Kremer, and L. Iftode. Smart Messages: A Distributed Computing Platform for Networks of Embedded Systems. The Computer Journal, Special Focus-Mobile and Pervasive Computing, 47(4):475-494, July 2004. The British Computer Society. Oxford University Press.

[12] S.-S. Kang and M. Mutka. Efficient mobile access to internet data via a wireless peer-to-peer network. In Proceedings of the Second IEEE International Conference on Pervasive Computing and Communications, pages 197-205, 2004.

[13] N. Karnik and A. Tripathi. Agent Server Architecture for the Ajanta Mobile-Agent System. In Proceedings of the 1998 International Conference on Parallel and Distributed Processing Techniques and Applications (PDPTA'98), pages 66-73, Las Vegas, NV, July 1998.

[14] J. Moore, M. Hicks, and S. Nettles. Practical Programmable Packets. In Proceedings of the 20th Annual Joint Conference of the IEEE Computer and Communications Societies (INFOCOM 2001), pages 41-50, Anchorage, AK, April 2001.

[15] H. Peine and T. Stolpmann. The Architecture of the Ara Platform for Mobile Agents. In First International Workshop on Mobile Agents MA'97, pages 50-61, 1997.

[16] M. Ranganathan, A. Acharya, S. Sharma, and J. Saltz. Network-aware Mobile Programs. In Proceedings of the USENIX 1997 Annual Technical Conference, pages 91-104, 1997.

[17] T. Sakamoto, T. Sekiguchi, and A. Yonezawa. Bytecode Transformation for Portable Thread Migration in Java. In ASA/MA, pages 16-28, 2000.

[18] E. Truyen, B. Robben, B. Vanhaute, T. Coninx, W. Joosen, and P. Verbaeten. Portable Support for Transparent Thread Migration in Java. In ASA/MA, pages 29-43, 2000. 